\title{
General health in infertile women with polycystic ovary syndrome under infertility treatment referred to Sarem hospital
}

\section{ARTICLE INFO}

\section{Article Type}

Original Article

\section{Authors}

Fariba Mohammad Aliha ${ }^{1}$, B.S

Mahtab Moti ${ }^{2}$, ID, MSc*

Leila Amini ${ }^{2}$, ID $\mathrm{PhD}^{*}$

Hamid Haghani ${ }^{3}, \mathrm{PhD}$

Homa Sadeghi Avval Shahr ${ }^{2}$, iD $\mathrm{PhD}$

Sarem Fertility \& Infertility Research Center (SAFIR) \& Sarem Cell Research Center (SCRC), Sarem Women's Hospital, Iran University of Medical Sciences (IUMS), Tehran, Iran.

2 Nursing and Midwifery School, Iran University of Medical Sciences, Tehran, Iran.

3 School of Public Health, Iran University of Medical Sciences, Tehran, Iran.

*Corresponding Author Address: School of Nursing \& Midwifery, Rashid Yasemi st.

Valiasr St., Tehran, IRAN. Postal code: 1996713883

Phone: +98 (21) 43651000

Fax: +98 (21) 88201978

\section{ABSTRACT}

Introduction: Infertility is one of the common problems in reproductive age and polycystic ovary syndrome (PCOS) is the most common cause of infertility. The aim of this study was to determine the general health of infertile women with PCOS referred to Sarem hospital.

Methods: This cross-sectional study was performed on 64 married women suffering PCOS with age 18 to 40 years undergoing infertility treatment. The data collection tools in this study included General Health Questionnaire (GHQ) and demographic information that its validity was confirmed by faculty members. Data were analyzed using SPSS software version 20 and the results were demonstrated in tables.

Results: The results of this study demonstrated that the mean age of subjects was 29.82 \pm 3.04 years and marriage duration was $12.98 \pm 2.99$ years and these patients had a history of $9.98 \pm 2.98$ years of infertility averagely. Most of these people had primary infertility $(93.7 \%)$ and others patients had secondary infertility (6.3\%). Also, in terms of general health, $43.7 \%$ of participants in this study had a mild disorder. The percentage of mild disorders in general health included $60.9 \%$ of physical symptoms, $37.6 \%$ of those with anxiety and sleep disorders, and $51.5 \%$ of social functions, while $68.8 \%$ had severe depression. But, none of these participants in the research had an intense disorder in the areas of social function. Conclusion: It can be concluded from this study that most infertile people under treatment had a severe disturbance in the depression; therefore, it seems that screening strategies and depression treatment should be noticed in their infertility course.

Keywords: Polycystic Ovary Syndrome (PCOS); Infertility; General Health; Depression; Anxiety; Social Functions; General Health Questionnaire (GHQ).

\section{Article History}

Received: December 05, 2020

Accepted: December 20, 2020

e Published: July 15, 2021

Copyright(C) 2021, ASP Ins. This open-access article is published under the terms of the Creative Commons Attribution-Noncommercial 4.0 International License which permits Share (copy and distribute the material in any medium or format) and Adapt (remix, transform, and build 


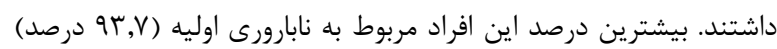

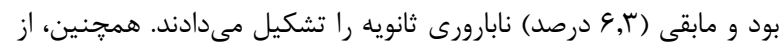

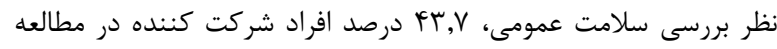

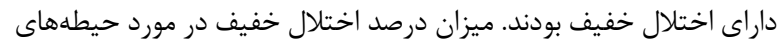

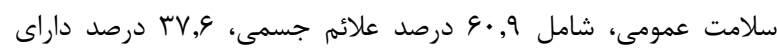

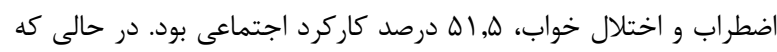

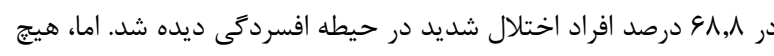
يك از افراد شركت كننده در يزوهش داراى اختلال شديد در حرد حيطه كار كارد

اجتماعى نبودند.

نتيجهَيرى: از اين مطالعه مى توان نتيجه كَرفت كه بيشترين تعداد افراد

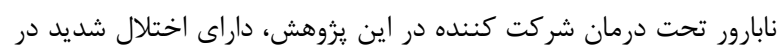

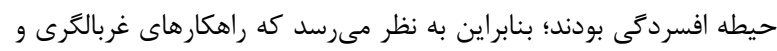

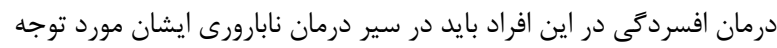

بيشترى قرار گيرد.

كليد وازمها: سندرم تخمدان يلى كيستيك؛ نابارورى؛ سلامت عمومى؛

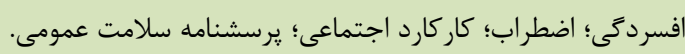

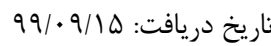

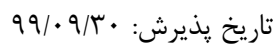

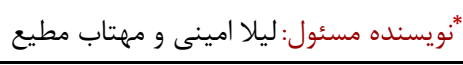

مقدمه

مشكل نابارورى در سراسر جهان، جوامع مختلف رادركير نموده و ييامدهاى

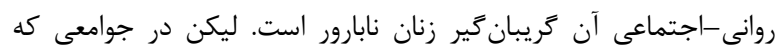

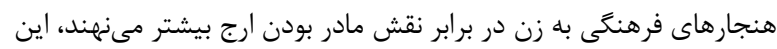

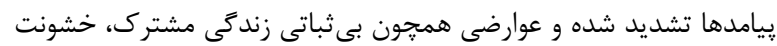

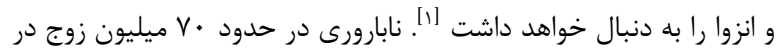

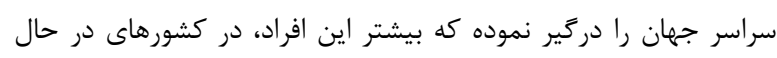

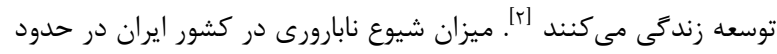

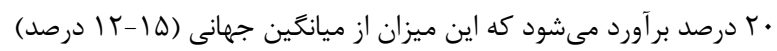

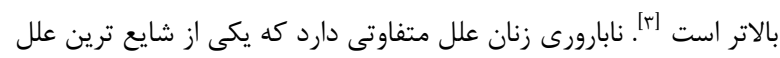
نابارورى سندرم تخمدان يلى كيستيك 'مى باشد.

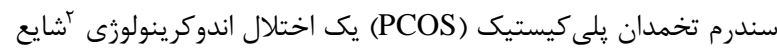

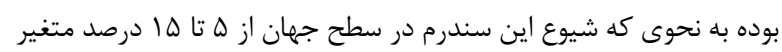

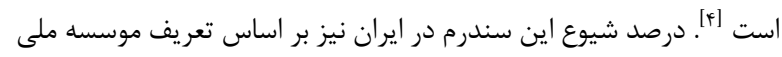

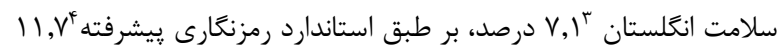

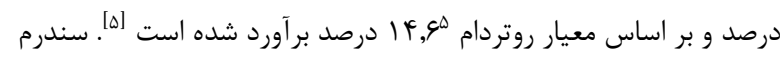

Advanced Encryption Standard (AES) Rotterdam Criteria ${ }^{\circ}$

\section{سلامت عمومى در زنان نابارور مبتلا} به سندرم تخمدان يلى تيستيك تحت درمان نابارورى مراجعه كننده به بيمارستان فوق تخصصى صارم

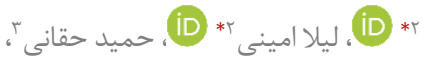

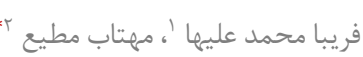
(iD) مما صادقى اول شهر

' مركز تحقيقات بارورى و نابارورى صارم، مركز تحقيقات سلولى و مولكولى

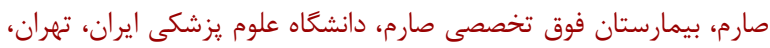

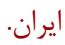

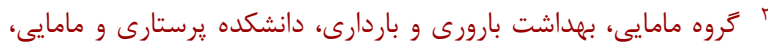
دانشخاه علوم يزشكى ايران.، تهران، ايران.

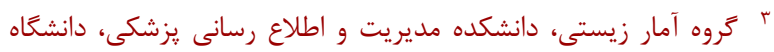
علوم يزشكى ايران.، تهران، ايران.

جكيده زمينه و هدف: نابارورى يكى از مشكلات شايع سنين بارورى است و سندرم تخمدان يلى كيستيك (PCOS) شايعترين علت نابارورى مى باشد.

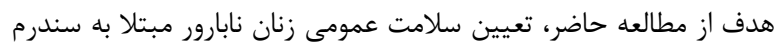

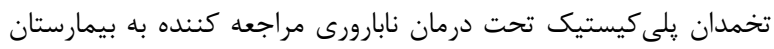
فوق تخصصى صارم بود.

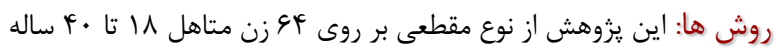

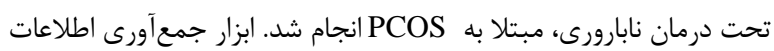

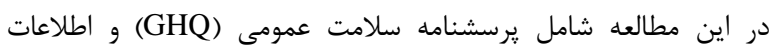
دموكر افيك بود كه روايى آن توسط اعضاى هيأت علمى تائيد كرديد. دادهها

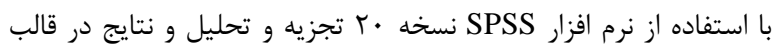
جداول ارائه شد. يافته ها: نتايج حاصل از اين مطالعه نشان داد كه ميانخين سن افراد مورد

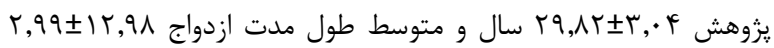

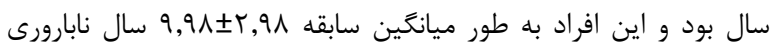

Polycystic Ovary Syndrome (PCOS) Endocrinologic Disorder National Institutes of Health (NIH) 
مشكل مىشوند [َ'] قرار ترفتن در سيكل درمانهاى مختلف نابارورى نيز

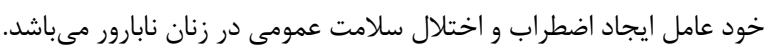

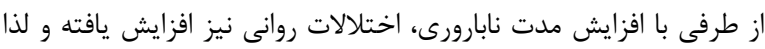

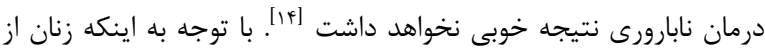

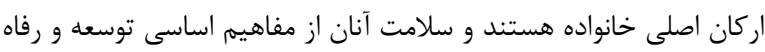

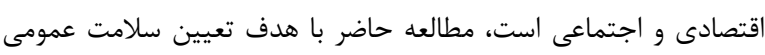

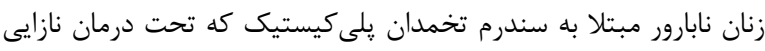
نيز مىباشند، انجام كرديد.

\section{روش اجرا}

يُروهشَّر پِ از كسب تائيديه از كميته اخلاق دانشَاه علوم يزشكى ايران

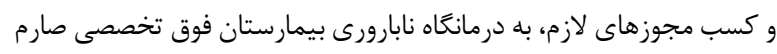

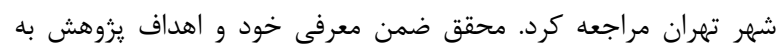

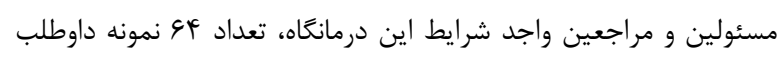

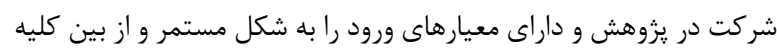

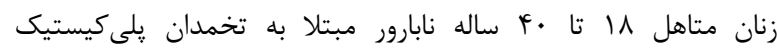

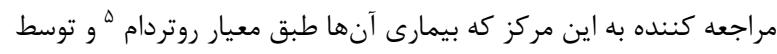

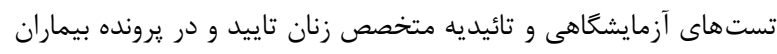

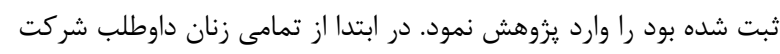

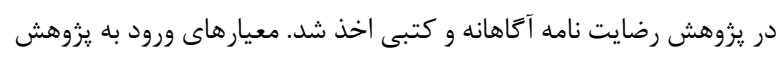

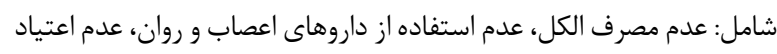

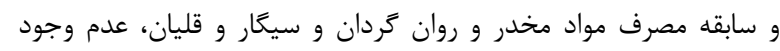

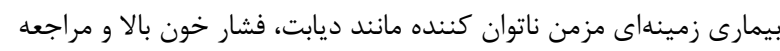

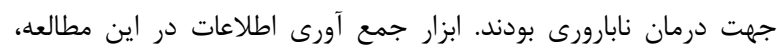

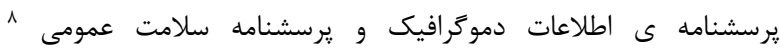
(GHQ-28) بر كيرنده جهار خرده مقياس (علائم جسمى، اختلال خواب و و اضطراب،

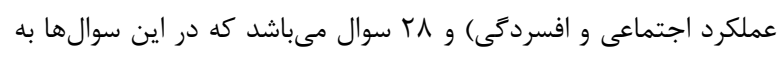

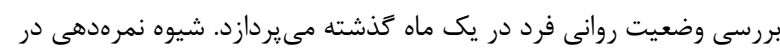

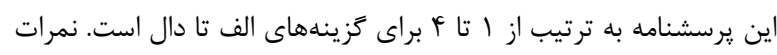

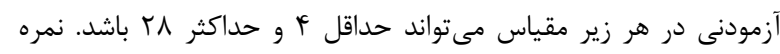

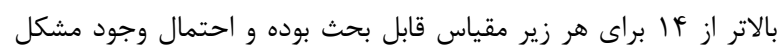

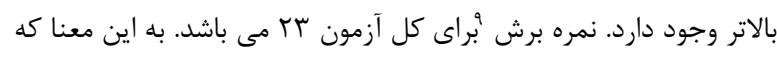

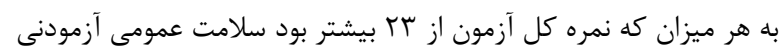

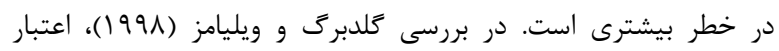

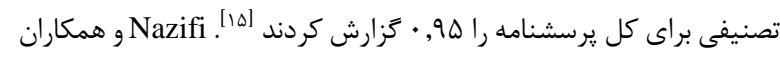

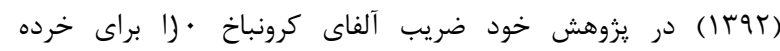
مقياسهاى علائم جسمانى، اضطراب و بى خونى خوابى، نارسايى در در عملكرد

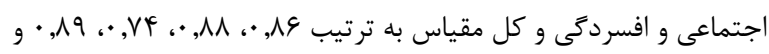

Cutoff Point ${ }^{9}$ Cronbach's Alpha
تخمدان يلى كيستيك اختلالى است كه مشخصه اصلى آن اختلال تخمك آند

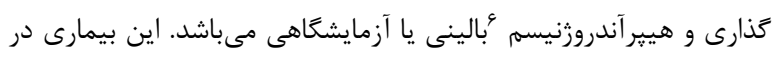
سنين مختلف كودكى (بلوغ زودرس)، نوجوانى (هيرسوتيسم و اختلال

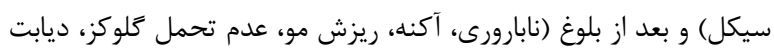

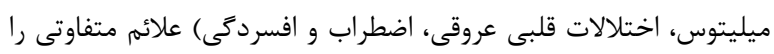

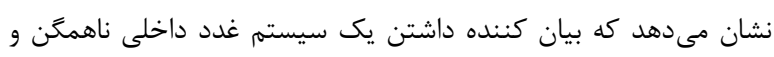

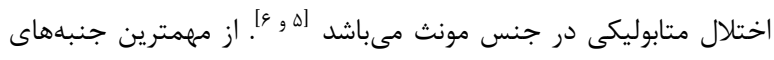

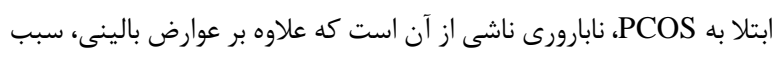

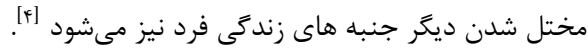

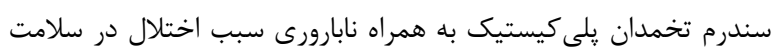

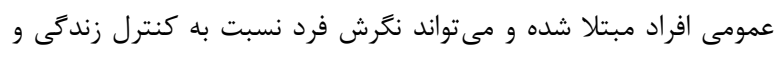

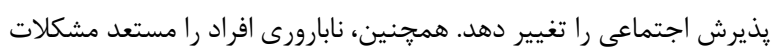

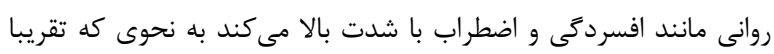

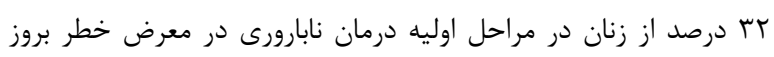

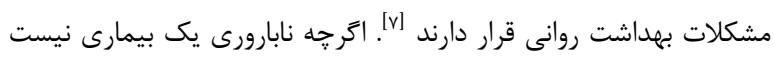

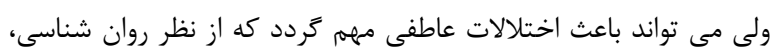

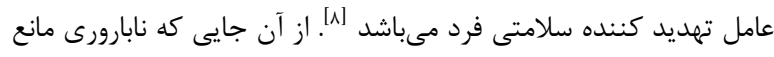

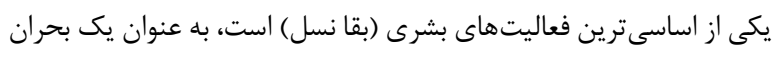

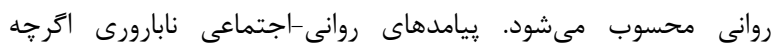

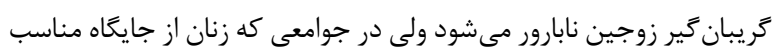

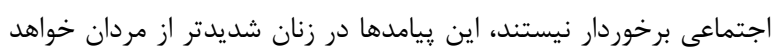

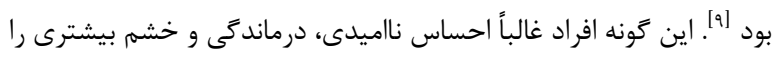

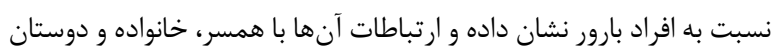
مختل شده و بيش از سايرين در معرض ابتلا به بيمارى هاى عاطفى -روانى، باري،

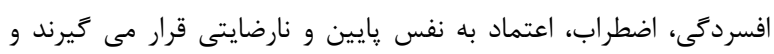

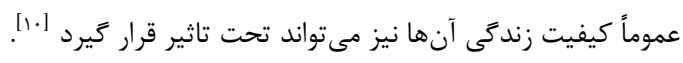

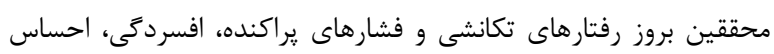

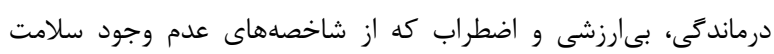

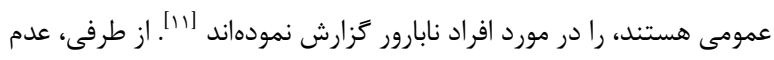

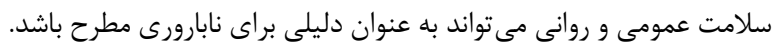

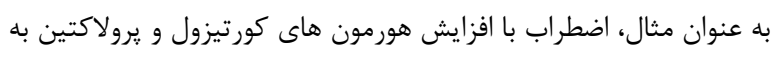
تشديد نابارورى مى انجامد. مكانيسمهاى متفاوتى در توجيه اين مسئله ارائه إنه

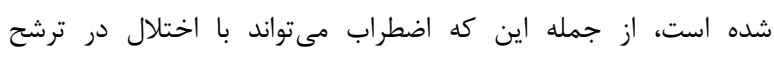

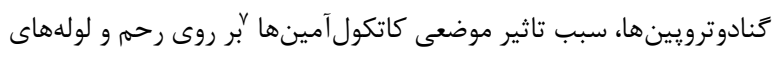

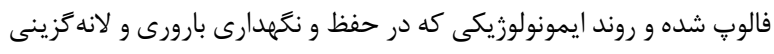

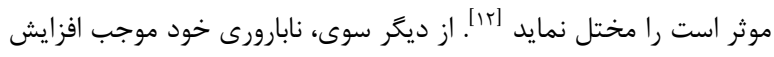

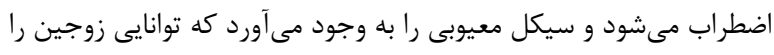

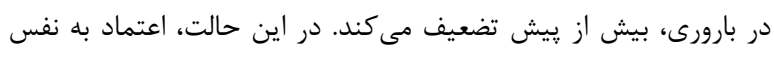

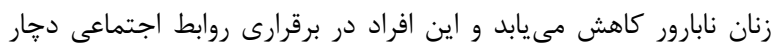




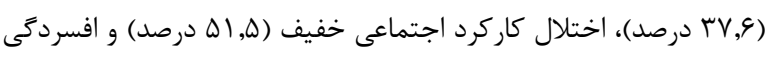

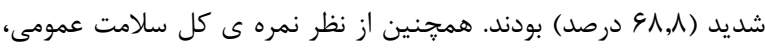

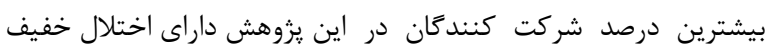
مطود ( F,V)

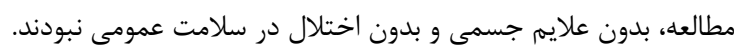

\begin{tabular}{|c|c|c|c|c|c|c|c|c|}
\hline \multicolumn{9}{|c|}{ جطالعه شماره - شاخص هاى عددى شدت اختلال نمرات كل و حيطه هاى سلامت عمومى زنان شركت كننده در } \\
\hline \multicolumn{2}{|c|}{ شديد } & \multicolumn{2}{|c|}{ متوسط } & \multicolumn{2}{|c|}{ خفيف } & \multicolumn{2}{|c|}{ هبج يا ممترين } & شدت اختلال \\
\hline درصد | د لمد & 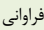 & درصد & فراوانى & درصد & فراوانى & درصد | لمد & 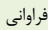 & \\
\hline$r, \Delta$ & 10 & 10,9 & 1. & $4 \cdot, 9$ & rq & $\cdot, \cdot$ & . & علائم جسمى \\
\hline $\mid \Lambda, V$ & ir & TQ & 19 & $r V, g$ & rf & $1 \Lambda, Y$ & ir & 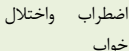 \\
\hline$\cdot, \cdot$ & . & $1 \cdot, 9$ & v & $\Delta 1, \Delta$ & זr & $r v, \varphi$ & rf & كار كرد اجتماعى \\
\hline $4 \wedge, \Lambda$ & fr & $1 \cdot, 9$ & v & $1 \cdot, 9$ & v & 9,4 & 9 & افسردىى \\
\hline$r r, \Delta$ & 10 & $r r, \Lambda$ & rI & Fr, $\mathrm{v}$ & ru & $\cdot, \cdot$ & . & نمره كل \\
\hline
\end{tabular}

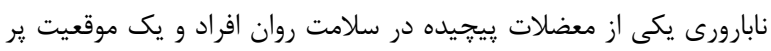

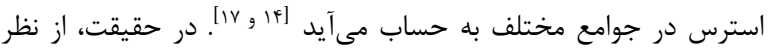

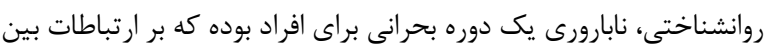

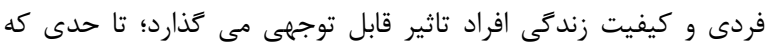

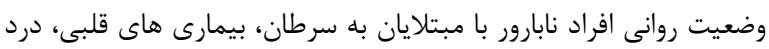

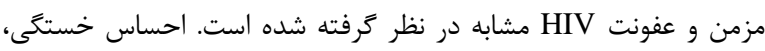

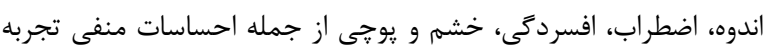

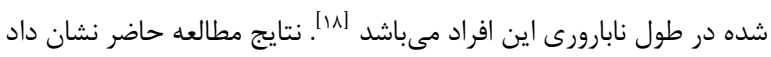

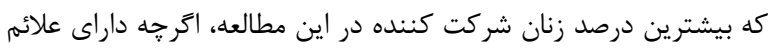

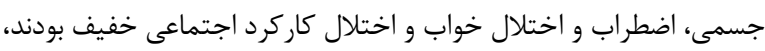

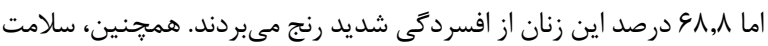

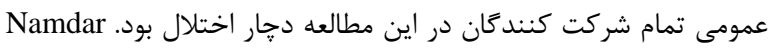

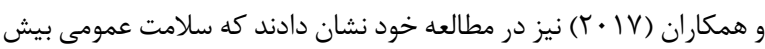

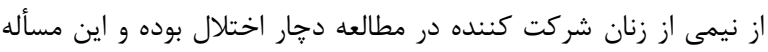

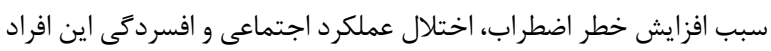

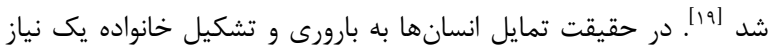

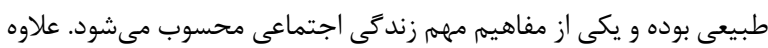

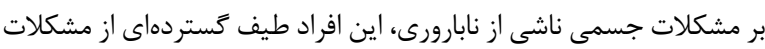

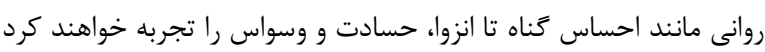

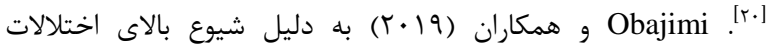

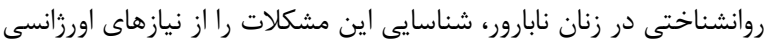

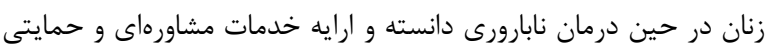

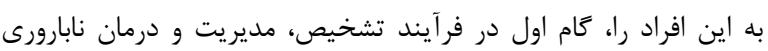

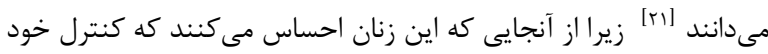

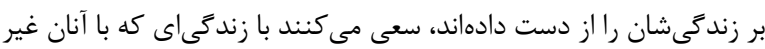

بو, • اعلام كردند [91]] دادههاى جمع آورى شده در اين يروهش، توسط

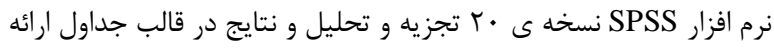

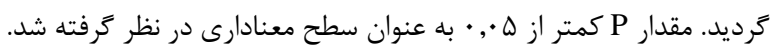

بافتته ها

در اين مطالعه تعداد \&4 زن نابارور مبتلا به تخمدان يلى كيستيك شركت

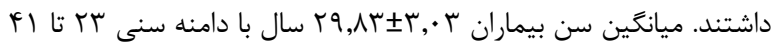

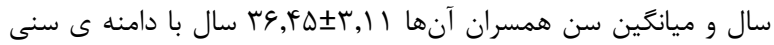

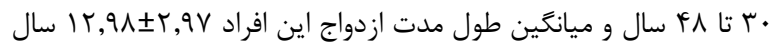

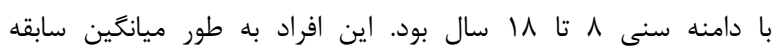
ناباورى

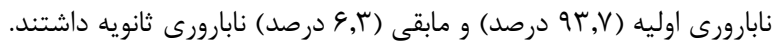

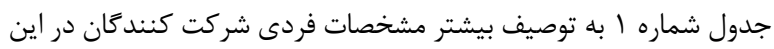

مطالعه مى يردازد.

\begin{tabular}{|c|c|c|}
\hline درصد & فراوانى & بارامتر \\
\hline $\begin{array}{l}1 \cdot, 9 \\
r \mu, \Lambda \\
r \Delta, r\end{array}$ & $\begin{array}{l}r \\
r \Delta \\
r q\end{array}$ & 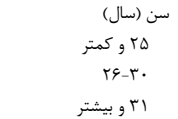 \\
\hline $\begin{array}{l}\mid \gamma, 1 \\
4 \Lambda, \Lambda \\
\mid f, 1\end{array}$ & $\begin{array}{l}11 \\
\text { ff } \\
9\end{array}$ & 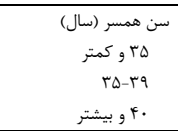 \\
\hline $\begin{array}{l}r r, \Delta \\
\Delta f, r \\
r r, \Lambda\end{array}$ & $\begin{array}{l}\hat{i} \\
r \Delta \\
r i\end{array}$ & 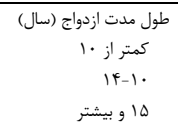 \\
\hline $\begin{array}{l}r 1, q \\
r \cdot, \varphi \\
r r, \Delta\end{array}$ & $\begin{array}{l}\text { if } \\
\text { is } \\
\text { if }\end{array}$ & 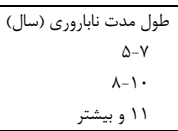 \\
\hline $\begin{array}{l}r \cdot, r \\
\Delta f, r \\
r \Delta, \cdot\end{array}$ & $\begin{array}{l}\text { ir } \\
\text { ro } \\
\text { is }\end{array}$ & 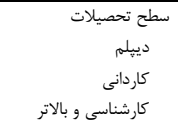 \\
\hline $\begin{array}{l}1 \Delta, \varphi \\
\Delta q, \uparrow \\
r \Delta, .\end{array}$ & $\begin{array}{l}1 . \\
\text { in } \\
19\end{array}$ & كارديلم تحصيلات همسر \\
\hline $\begin{array}{l}r \psi_{q, \digamma} \\
\varphi_{\Delta, \xi}\end{array}$ & $\begin{array}{l}r r \\
r\end{array}$ & وضعيت اشتغال 1 واغندار \\
\hline $\begin{array}{l}v 9,9 \\
r r, f\end{array}$ & $\begin{array}{l}\text { iq } \\
10\end{array}$ & وضعيت اشتغال همسر \\
\hline $\begin{array}{l}1 \cdot, 9 \\
v 9, \mathrm{v} \\
9, \mathrm{f}\end{array}$ & $\begin{array}{c}\text { v } \\
\text { D1 } \\
4\end{array}$ & وضامطلوبت اقتصادى \\
\hline
\end{tabular}

جدول شماره Y نشان دهنده فراوانى و درصد حيطههاى سلامت عمومى و

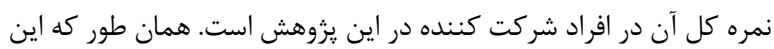

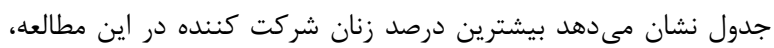

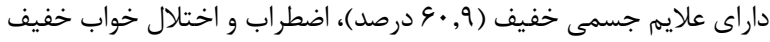




\author{
تاييديه اخلاقى

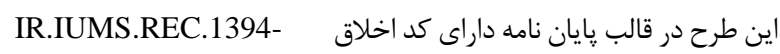

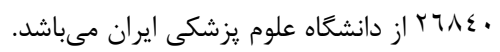 \\ تعارض منافع \\ در اين مطالعه هيج كونه تعارض منافعى وجود نداشت.

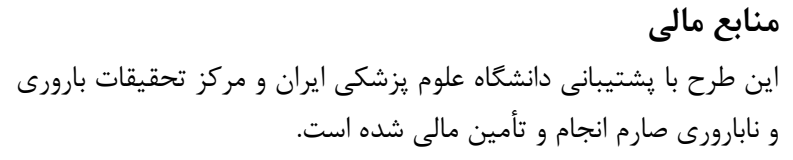

منابع

1. Vayena E, Rowe PJ, Griffin PD. Current practices and controversies in assisted reproduction: report of a meeting on medical, ethical and social aspects of assisted reproduction, held at WHO Headquarters in Geneva, Switzerland. World Health Organization; 2002.

2. Ombelet W, Cooke I, Dyer S, Serour G, Devroey P. Infertility and the provision of infertility medical services in developing countries. Hum Reprod Update. 2008;14(6):605-21.

3. Safdari R, Choobineh H, Rampisheh Z, Kameli ME, Vahedi BA. The need to design infertility registry in Iran. 2016;

4. Cunha A, Póvoa AM. Infertility management in women with polycystic ovary syndrome: a review. Porto Biomed J. 2021;6(1).

5. Sayehmiri F, Kiani F, Maleki F, Ahmadi M, Shohani M. Prevalence of polycystic ovary syndrome in Iranian women: a systematic review and metaanalysis. Iran J Obstet Gynecol Infertil. 2014;17(115):11-21.

6. Gnanadass SA, Prabhu YD, Gopalakrishnan AV. Association of metabolic and inflammatory markers with polycystic ovarian syndrome (PCOS): an update. Arch Gynecol Obstet. 2021;113.

7. Hussain A, Chandel RK, Ganie MA, Dar MA, Rather $\mathrm{YH}$, Wani ZA, et al. Prevalence of psychiatric disorders in patients with a diagnosis of polycystic ovary syndrome in Kashmir. Indian J Psychol Med. 2015;37(1):66-70.

8. Li G, Jiang Z, Kang X, Ma L, Han X, Fang M. Trajectories and predictors of anxiety and
عادلانه برخورد كرده است، بجنكَند. بنابراين، حمايت و مشاوره روانى زنان

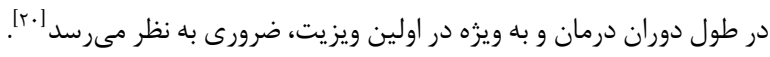

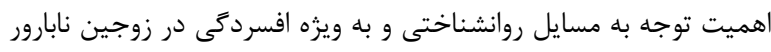

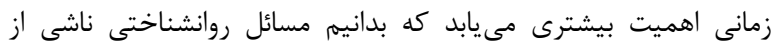

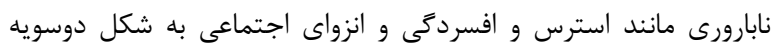

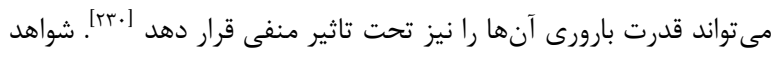

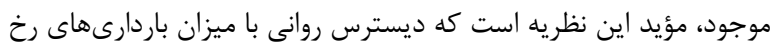

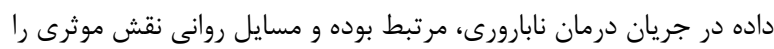

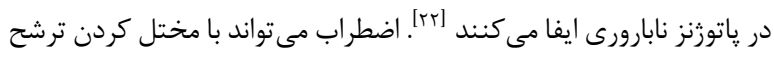

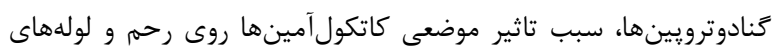

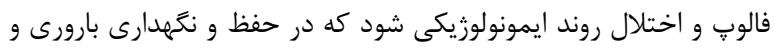

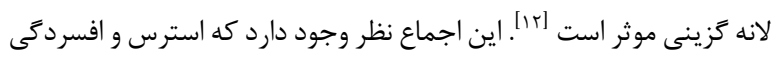

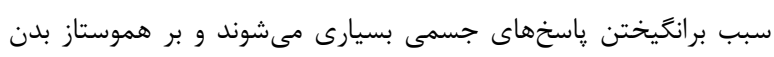

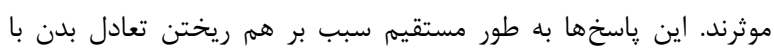

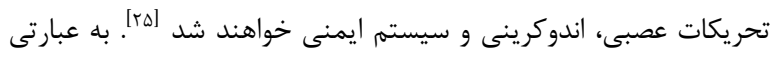

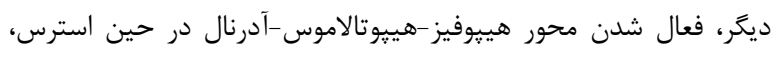

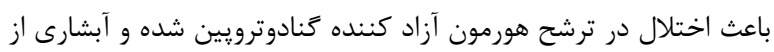

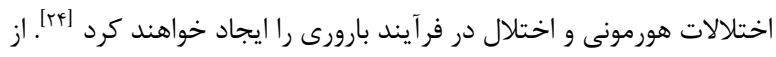
محدوديتهاى اين مطالعه مى توان به حجم كم نمونه اشاره كرد كه بهريتر

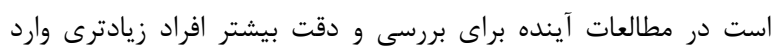
مطالعه شوند.

$$
\text { نتيجه گيرى }
$$

هدف از يزوهش حاضر، تعيين سلامت عمومى زنان نابارور مبتلا به سندرم

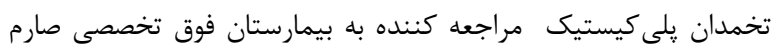

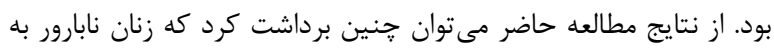

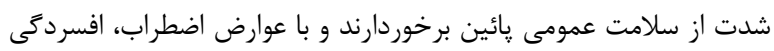

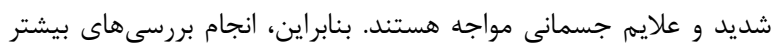

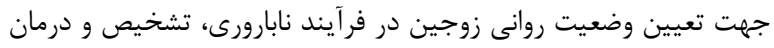

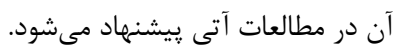

\section{تشكر و قدردانى}

اين كار بخشى از پايان نامه مقطع كارشناسى ارشد مى وباشد كه به طور

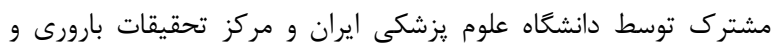

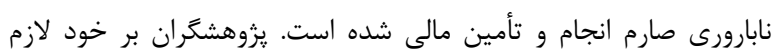

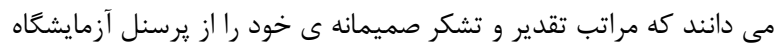

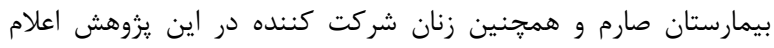

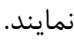


and anxiety disorders amongst a cohort of infertile women attending an in-vitro fertilization clinic in south-western Nigeria. Med J Zambia. 2019;46(3):192-6.

22. Deka PK, Sarma S. Psychological aspects of infertility. Br J Med Pract. 2010;3(3):336.

23. Cousineau TM, Domar AD. Psychological impact of infertility. Best Pract Res Clin Obstet Gynaecol. 2007;21(2):293-308.

24. Boivin J, Schmidt L. Infertility-related stress in men and women predicts treatment outcome 1 year later. Fertil Steril. 2005;83(6):1745-52.

25. Palomba S, Daolio J, Romeo S, Battaglia FA, Marci R, La Sala GB. Lifestyle and fertility: the influence of stress and quality of life on female fertility. Reprod Biol Endocrinol. 2018;16(1):1-11. depression amongst infertile women during their first IVF/ICSI treatment cycle. J Psychosom Res. 2021;142:110357.

9. Ching HL, Burke V, Stuckey BGA. Quality of life and psychological morbidity in women with polycystic ovary syndrome: body mass index, age and the provision of patient information are significant modifiers. Clin Endocrinol (Oxf). 2007;66(3):373-9.

10. Amini L, Ghorbani B, Afshar B. The Comparison of Infertility Stress and Perceived Social Support in Infertile Women and Spouses of Infertile Men. Iran J Nurs. 2020;32(122):74-85.

11. Sepidarkish M, Almasi-Hashiani A, Shokri F, Vesali S, Karimi E, Samani RO. Prevalence of infertility problems among Iranian infertile patients referred to Royan Institute. Int J Fertil Steril. 2016;10(3):278.

12. Teede HJ, Hutchison SK, Zoungas S. The management of insulin resistance in polycystic ovary syndrome. Trends Endocrinol Metab. 2007;18(7):273-9.

13. El Kissi Y, Romdhane A Ben, Hidar S, Bannour S, Idrissi KA, Khairi $H$, et al. General psychopathology, anxiety, depression and selfesteem in couples undergoing infertility treatment: a comparative study between men and women. Eur J Obstet Gynecol Reprod Biol. 2013;167(2):185-9.

14. Rooney KL, Domar AD. The relationship between stress and infertility. Dialogues Clin Neurosci. 2018;20(1):41.

15. Goldberg DP, Hillier VF. A scaled version of the General Health Questionnaire. Psychol Med. 1979;9(1):139-45.

16. Nazifi M, Mokarami HR, Akbaritabar AK, Faraji Kujerdi M, Tabrizi R, Rahi A. Reliability, validity and factor structure of the persian translation of general health questionnire (ghq-28) in hospitals of kerman university of medical sciences. J Fasa Univ Med Sci. 2014;3(4):336-42.

17. Ngai F-W, Loke AY. Relationships between infertility-related stress, family sense of coherence and quality of life of couples with infertility. Hum Fertil. 2021;1-13.

18. Aslzaker M, Pourshahbaz A, Bagheri Lankarani N, Mohammadkhani P, Geranmayepour S. Effects of infertility stress, psychological symptoms, and quality of life on predicting success rate of IVF/ICSI treatment in infertile women. Pract Clin Psychol. 2016;4(4):275-81.

19. Namdar A, Naghizadeh MM, Zamani M, Yaghmaei F, Sameni MH. Quality of life and general health of infertile women. Health Qual Life Outcomes. 2017;15(1):1-7.

20. Ramazan ZF, Nourbala AA, Abedinia N, Naghizadeh MM. Emotional adjustment in infertile couples. 2009;

21. Obajimi GO, Esan O, Ogunkinle BN. Depression 